\title{
Gamma-Delta T-Cell Depletion
}

National Cancer Institute

\section{Source}

National Cancer Institute. Gamma-Delta T-Cell Depletion. NCI Thesaurus. Code C131671.

Restriction of the T-cell population in stem cell transplants to those expressing only the alpha-beta receptor. 